\title{
Curvature-induced symmetry breaking in nonlinear Schrodinger models
}

\author{
Gaididei, Yuri Borisovich; Mingaleev, S. F.; Christiansen, Peter Leth
}

Published in:

Physical Review E. Statistical, Nonlinear, and Soft Matter Physics

Link to article, DOI:

10.1103/PhysRevE.62.R53

Publication date:

2000

Document Version

Publisher's PDF, also known as Version of record

Link back to DTU Orbit

Citation (APA):

Gaididei, Y. B., Mingaleev, S. F., \& Christiansen, P. L. (2000). Curvature-induced symmetry breaking in nonlinear Schrodinger models. Physical Review E. Statistical, Nonlinear, and Soft Matter Physics, 62(1), R53R56. https://doi.org/10.1103/PhysRevE.62.R53

\section{General rights}

Copyright and moral rights for the publications made accessible in the public portal are retained by the authors and/or other copyright owners and it is a condition of accessing publications that users recognise and abide by the legal requirements associated with these rights.

- Users may download and print one copy of any publication from the public portal for the purpose of private study or research.

- You may not further distribute the material or use it for any profit-making activity or commercial gain

- You may freely distribute the URL identifying the publication in the public portal

If you believe that this document breaches copyright please contact us providing details, and we will remove access to the work immediately and investigate your claim 


\title{
Curvature-induced symmetry breaking in nonlinear Schrödinger models
}

\author{
Yu. B. Gaididei and S. F. Mingaleev \\ Bogolyubov Institute for Theoretical Physics, 03143 Kiev, Ukraine \\ P. L. Christiansen \\ Department of Mathematical Modelling, The Technical University of Denmark, DK-2800 Lyngby, Denmark
}

(Received 25 February 2000)

\begin{abstract}
We consider a curved chain of nonlinear oscillators and show that the interplay of curvature and nonlinearity leads to a symmetry breaking when an asymmetric stationary state becomes energetically more favorable than a symmetric stationary state. We show that the energy of localized states decreases with increasing curvature, i.e., bending is a trap for nonlinear excitations. A violation of the Vakhitov-Kolokolov stability criterion is found in the case where the instability is due to the softening of the Peierls internal mode.

PACS number(s): 05.45.Yv, 33.15.Bh, 42.65.Tg, 63.20.Pw
\end{abstract}

Nanoscale physical systems such as biological macromolecules, nanotubes, microtubules, vesicles, and electronic and photonic waveguide structures [1-6] are usually objects with nontrivial geometry. The gene transcription is usually accompanied by a local DNA bending [7-9]; the electronic properties of carbon nanotubes drastically depend on their chirality [10]; $T$-shaped junctions were recently proposed [11] as nanoscale metal-semiconductor-metal contact devices. The geometry manifests itself in particular in the creation of linear quasibound states (see, e.g., $[12,13])$. On the other hand, the competition between nonlinearity and dispersion likewise results into localization of energy. Taken together these two localization mechanisms are competitive and one might expect that the interplay of geometry and nonlinearity can lead to qualitatively new effects. An example of nonlinearity-induced change of the geometry of the system is found in Ref. [14], where the classical Heisenberg model on a two-dimensional manifold was considered. It was shown that a periodic topological spin soliton induces a periodic pinch of the cylindric manifold.

In this Rapid Communication we consider nonlinear Schrödinger (NLS) models on a curved one-dimensional manifold and show that the competition between curvature and nonlinearity leads to another qualitative effect: curvature-induced symmetry breaking for nontopological solitary excitations. This effect is inherent for both continuum and discrete NLS models. We start with the discrete NLS equation

$$
i \frac{d}{d t} \psi_{n}+\sum_{m} J_{n m} \psi_{m}+\left|\psi_{n}\right|^{2} \psi_{n}=0
$$

which describes a system of coupled nonlinear oscillators, $\psi_{n}(t)$ being the complex amplitude at the site $n(n=0, \pm 1$, \pm 2 ). In the theory of charge (energy) transfer $\psi_{n}(t)$ is the wave function of the carriers. The Hamiltonian

$$
H=-\sum_{n}\left\{\sum_{m} J_{n m} \psi_{n}^{*} \psi_{m}+\frac{1}{2}\left|\psi_{n}\right|^{4}\right\}
$$

and the number of excitations (quanta) $N=\Sigma_{n}\left|\psi_{n}\right|^{2}$ are conserved quantities. In Eq. (1) the nonlinear term $\left|\psi_{n}\right|^{2} \psi_{n}$ rep- resents a self-interaction of the quasiparticle (anharmonicity of vibrations). The excitation transfer $J_{n m}$ in the coupling term depends on the distance in the embedding space between the sites $n$ and $m: J_{n m} \equiv J\left(\left|\vec{r}_{n}-\vec{r}_{m}\right|\right)$, where the radiusvector $\vec{r}_{n}=\left(x_{n}, y_{n}, z_{n}\right)$ characterizes the position of the site $n$ on the manifold. The sites are assumed to be equidistantly placed on a plane envelope curve (Fig. 1). This model is closely related to the so-called wormlike inextensible chain model $[15,16]$ which is abundantly used in polymer dynamics (see, e.g., [17-19]). We consider the properties of nonlinear excitations in the vicinity of a smooth bend where the curve can be modeled by the parabola without loss of generality (as done in Fig. 1): $y_{n}=\kappa x_{n}^{2} / 2$ and $z_{n}=0$, with $\kappa$ being the inverse radius of curvature at the bending point. The distance between neighboring sites is assumed to be independent of the curvature $\kappa:\left|\vec{r}_{n+1}-\vec{r}_{n}\right|=1$ (inextensibility of the chain). Therefore, the excitation dynamics would not depend on the curvature of the system in the nearestneighbor approximation, when $J_{n m}=J \delta_{n-m, \pm 1}$. In many physical problems, however, the nearest-neighbor approximation is too crude. For example, the DNA molecule contains charged groups, and therefore the vibration-excitation transfer is due to the dipole-dipole interaction decaying with the distance $r$ as $1 / r^{3}$. Effective dispersive interaction between nonlinear layers in nonlinear dielectric superlattices is exponential, $\exp (-\alpha r)$, with the inverse radius $\alpha$ depending

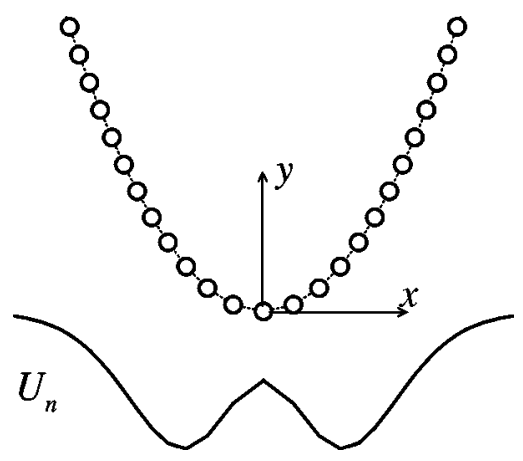

FIG. 1. Curved chain with sites equidistantly placed on a parabola with the curvature $\kappa=0.5$ at $x=0$ and corresponding effective double-well potential $U_{n}=-\Sigma_{m} J_{n, m}$. 


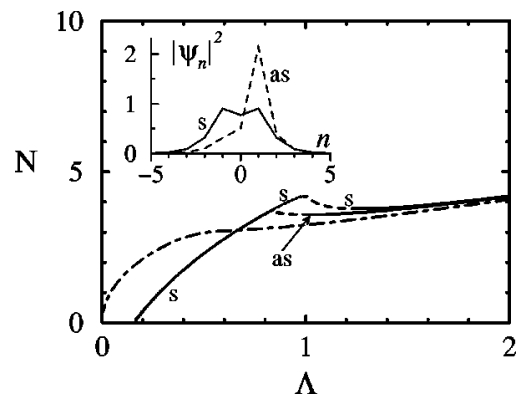

FIG. 2. Number of excitations $N(\Lambda)$ for the straight chain $(\kappa$ $=0$; dotted-dashed line) and curved chain ( $\kappa=3$; solid, dotted, and dashed lines) for $\alpha=2$ and $J=6.4$. In the inset shapes of the symmetric $(s)$ and asymmetric (as) stationary states for $N=3.6$ are shown.

on parameters of the lattice (superlattice spacing, linear refractive index and so on) [20]. When the long-range character of the coupling $J_{n m}$ is considered, the number of neighbors available for the excitation is larger near the bending point than far away from it. This creates (as shown below analytically) the effective double-well potential $U(\ell)$, which is appreciably responsible for the effects discussed in what follows.

We investigated both $J_{n m}=J \exp \left(-\alpha\left|\vec{r}_{n}-\vec{r}_{m}\right|\right)$ and $J_{n m}$ $=J\left|\vec{r}_{n}-\vec{r}_{m}\right|^{-s}$. But in this Rapid Communication we discuss in detail the first case only. It is known [21] that in the case of straight line $(\kappa=0)$ such NLS model exhibits (for $\alpha$ $<1.7$ ) bistability in the spectrum of nonlinear stationary states. To distinguish these effects from the finite curvature effects we use $\alpha=2$. We study stationary states of the system $\psi_{n}(t)=\phi_{n}(\Lambda) e^{i \Lambda t}$, where $\Lambda$ is the nonlinear frequency and $\phi_{n}(\Lambda)$ is the amplitude in the $n$th site.

Figure 2 shows $N$ versus $\Lambda$ obtained numerically for the straight chain and curved chain. Several new features arise as a consequence of the finite curvature:

(i) There is a gap at small $\Lambda$ in which no localized excitation can exist. The gap originates from the existence, in the linear case, of a two-hump localized mode created by curvature. The gap increases when the curvature $\kappa$ increases.

(ii) There are two branches of stationary states: a branch of symmetric localized excitations $(s)$, which exists for all values of $N$, and a branch of asymmetric localized excitations (as), which exists only for $N>N_{t h}(\kappa)$. The threshold value $N_{t h}(\kappa)$ decreases when $\kappa$ decreases and vanishes in the limit $\kappa \rightarrow 0$. The symmetric stationary state has a two-humped shape (evolved from the linear two-hump localized mode), while in the asymmetric state the maximum is shifted either to the left or to the right from the center of symmetry $n=0$ (see inset in Fig. 2).

(iii) For the symmetric stationary states $N(\Lambda)$ is nonmonotonic as in Refs. [22,21] for NLS models on the straight chain with the radius of the dispersive interaction above a critical value. Similarly, in the case of curved chain there is an interval of $N$ in which three different symmetric states coexist for each excitation number. Taking into account that for the inverse radius $\alpha=2$ under investigation there is no bistability in the straight chain (see dotted-dashed line in Fig. 2 ), one may conclude that bistability is facilitated in the systems with finite curvature.

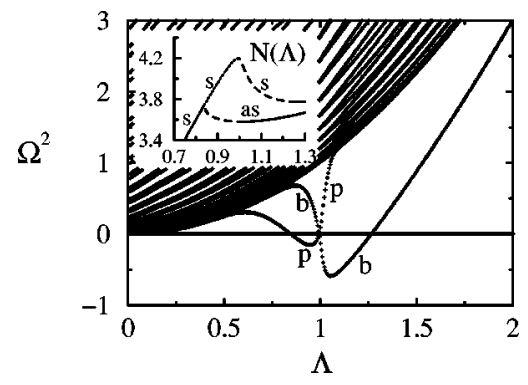

FIG. 3. Spectrum of linear excitations around the symmetric stationary state $\phi_{n}(\Lambda)$ for $\alpha=2, J=6.4$, and $\kappa=3$. There are two internal localized modes: Peierls antisymmetric $(p)$ and breathing symmetric $(b)$. In the inset the dependence $N(\Lambda)$ is shown.

The linear stability of the stationary states is investigated (as is described in Refs. [23,21]), letting $\psi_{n}(t)=\left[\phi_{n}(\Lambda)\right.$ $\left.+\epsilon_{n} \exp (i \Omega t)\right] \exp (i \Lambda t)$ and linearizing Eq. (1) with respect to $\epsilon_{n}$. By definition the stationary state $\phi_{n}(\Lambda)$ is linearly stable if $\Omega^{2}>0$. Figure 3 shows the spectrum of linear excitations around the symmetric localized state. We find that there are two localized internal modes: Peierls antisymmetric mode $(p)$ and breathing symmetric mode $(b)$. There are two intervals of instability of the symmetric stationary state: when $0.99<\Lambda<1.26$ the instability is due to the breathing internal mode. In this part of the curve $N(\Lambda)$ (dashed line in the inset) $d N / d \Lambda<0$ and the instability agrees with the Vakhitov-Kolokolov stability criterion [24]. When $0.84<\Lambda$ $<0.99$ the stationary state is unstable with respect to the Peierls mode (the corresponding part of $N(\Lambda)$ is marked by the dotted line). In this latter case we observe a violation of the Vakhitov-Kolokolov stability criterion because this instability interval corresponds to a part of the curve $N(\Lambda)$ where $d N / d \Lambda>0$ (see Fig. 2).

The energy of the symmetric as well as asymmetric localized states is monotonically decreasing function of the curvature (see Fig. 4). Thus, one may expect that the nonlinear localized excitation may facilitate bending of flexible molecular chain. For $N>N_{t h}(\kappa)$, when symmetric and asymmetric stationary states coexist, the asymmetric state is always energetically more favorable. Taking into account that in the linear limit the ground state of the model is symmetric with respect to the center of symmetry $n=0$ one can conclude that combined action of the finite curvature and nonlinearity provides the symmetry breaking in the system. A typical evolution of the curved NLS model in the case when the symmetric stationary state becomes unstable is shown in Fig. 5. One can see that the two-humped initial symmetric state evolves into an asymmetric state with an excited breathing internal mode.

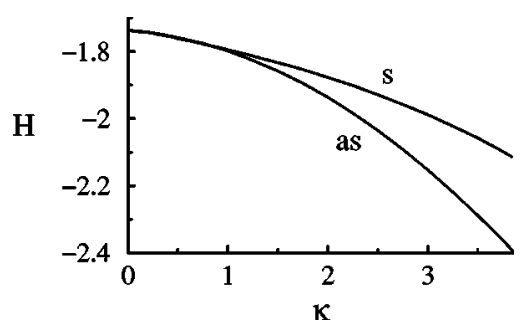

FIG. 4. Energy of symmetric ( $s$ ) and asymmetric (as) nonlinear excitations vs the curvature $\kappa$ for $\alpha=2, J=6.4$, and $N=4$. 


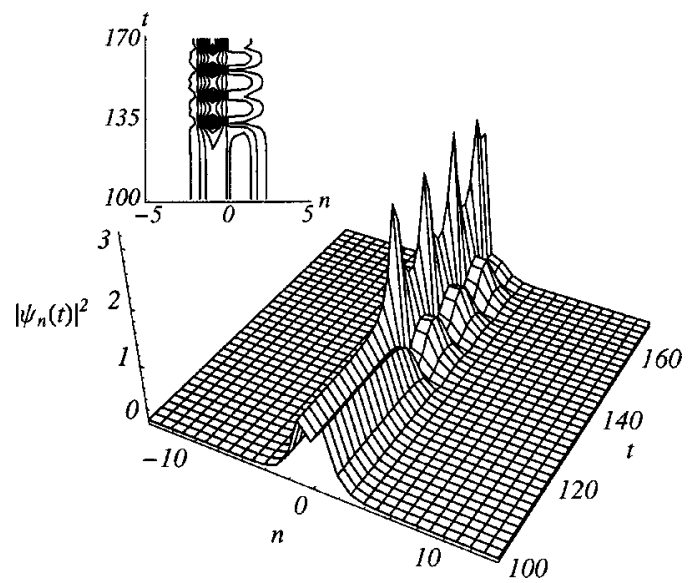

FIG. 5. Switching from the symmetric state to the asymmetric state at $\alpha=2, J=6.4, \kappa=4$, and $N=3.96$.

Apart from the smooth bending we studied the case of the edge: $y_{n}=\left|x_{n}\right| \tan (\theta)$. Qualitatively the same results were obtained for this case, too.

To clarify the physical meaning of obtained results we pass to the continuum limit of Eq. (2), where the Hamiltonian $H$ can be represented as

$$
H=\int_{-\infty}^{\infty}\left(\frac{1}{2} L^{2}(\ell)\left|\frac{\partial \psi}{\partial \ell}\right|^{2}+U(\ell)|\psi|^{2}-\frac{1}{2}|\psi|^{4}\right) d \ell .
$$

Here $\ell$ is the arclength (the continuum analog of $n$ ), $L^{2}(\ell)=\int_{-\infty}^{\infty}\left(\ell^{\prime}-\ell\right)^{2} J_{\ell, \ell^{\prime}} d \ell^{\prime}$ is the square of the dispersive length of the excitation, and $U(\ell)=-\int_{-\infty}^{\infty} J_{\ell, \ell^{\prime}} d \ell^{\prime}$ is the energy shift due to the coherent interaction. The inverse length $L^{-1}(\ell)$ and attractive potential $U(\ell)$ are generally double-well functions. At the bottom of Fig. 1 the discrete analogue of $U(\ell), U_{n}=-\Sigma_{m} J_{n, m}$, is shown. In the vicinity of the bend there are two symmetric sections of the chain in which the number of neighbors entering into the range of the dispersive interaction, $1 / \alpha$, is higher than anywhere else on the chain. In this way a double-well potential structure results. The positions and depths of the wells are determined by the range of the dispersive interaction and the peculiarities of the bending geometry. For example, in the case of the edge, $y_{n}=\left|x_{n}\right| \tan (\theta)$, we get for small $\theta$

$$
L^{2}(\ell) \approx \frac{J}{\alpha^{3}}\left\{4+\left[\left(\alpha^{2}|\ell|+1\right)^{2}+1\right]\left(e^{-\alpha|\ell| \cos (2 \theta)}-e^{-\alpha|\ell|}\right)\right\}
$$

and

$$
U(\ell) \approx-\frac{J}{\alpha}\left(2+e^{-\alpha|\ell| \cos (2 \theta)}-e^{-\alpha|\ell|}\right) .
$$

It is worth noting that the Hamiltonian similar to Eq. (3) with $U(\ell) \equiv 0$ was introduced in Ref. [25] to study the effects of the DNA bending on breather trapping. The step function dependence of the dispersive length $L(\ell)$ was postulated there. In all cases bending manifests itself as a trap for nonlinear excitations. To obtain an analytical solution of the problem one can approximate $L(\ell)$ by a constant and $U(\ell)$ by two $\delta$-functions. Then we arrive at the following minimal model:

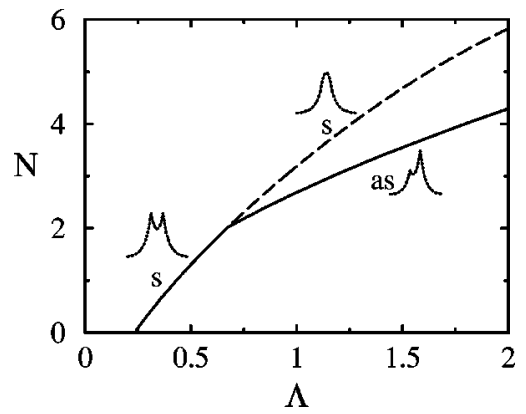

FIG. 6. Dependence $N(\Lambda)$ for symmetric $(s)$ and asymmetric (as) nonlinear excitations in the two-impurity minimal model (4) with $\epsilon=0.7$ and $a=1$.

$$
i \frac{\partial \psi}{\partial t}+\frac{\partial^{2} \psi}{\partial \ell^{2}}+\epsilon[\delta(\ell-a)+\delta(\ell+a)] \psi+|\psi|^{2} \psi=0
$$

where the parameter $\epsilon$ is the depth of the well. Introducing into Eq. (4) the stationary state ansatz $\psi(\ell, t)$ $=\phi(\ell) \exp (i \Lambda t)$ we find that the corresponding nonlinear eigenvalue problem can be easily reduced to an algebraic one. The results of the analytical consideration are shown in Fig. 6 . The stationary state $(s)$ is unique and symmetric for small number of quanta $N$. But when the number of quanta exceeds some critical value there are two stationary states: symmetric $(s)$ and asymmetric (as), with the asymmetric state being energetically more favorable. Thus, similarly to the original model, the minimal model demonstrates a symmetry breaking effect (localization in one of the wells).

We conclude that in the NLS model the interplay of curvature and nonlinearity leads to the symmetry breaking when the asymmetric stationary state becomes energetically favorable than the symmetric one. We have found that the energy of localized states decreases with the increasing of curvature. One may expect that the nonlinear localized excitation may facilitate bending of a flexible molecular chain. In all cases bending manifests itself as a trap for nonlinear excitations. For the curved NLS model in the case of the physically important dipole-dipole dispersive interaction $J_{n m}$ $=\left|\vec{r}_{n}-\vec{r}_{m}\right|^{-3}$ qualitatively the same properties are found. Using as an example the Davydov-Scott model [26,27] with the dipole-dipole coupling between sites [28] for the parameters which characterize the motion of the amide-I excitation in proteins (they correspond to $N=0.64$ in our model) decreasing of the ground state energy due to a trapping by the bending with $\kappa=1 \quad(\kappa=3)$ comprise $10 \%(46 \%)$ of the whole excitation energy. This may also be important for the dynamics of DNA molecule, where, as was mentioned above, the vibration-excitations are described by Eq. (1) with the dipole-dipole dispersive interaction [29]. Finally, a violation of the Vakhitov-Kolokolov stability criterion in the case when the instability is due to the softening of the Peierls internal mode is found.

We thank A. C. Scott for helpful discussions. Yu.B.G. and S.F.M. would like to express their thanks to the Department of Mathematical Modelling, Technical University of Denmark, where the major part of this work was done. 
[1] W. Saenger, Principles of Nuclear Acid Structure (SpringerVerlag, Berlin, 1984).

[2] S. Iijima, Nature (London) 354, 56 (1991).

[3] R. Bar-Ziv and E. Moses, Phys. Rev. Lett. 73, 1392 (1994); P. Nelson, T. Powers, and U. Seifert, ibid. 74, 3384 (1995).

[4] Physics of Amphiphilic Layers, edited by J. Meunier, D. Langevin, and N. Boccara (Springer-Verlag, Berlin, 1987).

[5] Y. Imry, Introduction to Mesoscopic Physics (Oxford University Press, New York, 1997).

[6] Photonic Band Gaps and Localization, edited by C. M. Soukoulis (Plenum Press, New York, 1993).

[7] C. Reiss, in Nonlinear Excitations in Biomolecules, edited by M. Peyrard (Springer, Berlin, Les Editions de Physique, Les Ullis, 1995), p. 29.

[8] H. Heumann, M. Ricchetti, and W. Werel, EMBO J. 7, 4379 (1988)

[9] S. K. Burley, Nature Struct. Biol. 1, 638 (1994); J. L. Kim, D. B. Nikolov, and S. K. Burley, Nature (London) 365, 520 (1993).

[10] N. Hamada, S. Sawada, and A. Oshiyama, Phys. Rev. Lett. 68, 1579 (1992).

[11] M. Menon and D. Srivastava, Phys. Rev. Lett. 79, 4453 (1997).

[12] G. Kirczenow, Phys. Rev. B 39, 10452 (1989).

[13] Yu. B. Gaididei, L. I. Malysheva, and A. I. Onipko, J. Phys.: Condens. Matter 4, 7103 (1992).

[14] R. Dandoloff, S. Villain-Guillot, A. Saxena, and A. R. Bishop,
Phys. Rev. Lett. 74, 813 (1995); A. Saxena and R. Dandoloff, Phys. Rev. B 55, 11049 (1997).

[15] O. Kratky and G. Porod, Recl. Trav. Chim. Pays-Bas. 68, 1106 (1949).

[16] N. Saitô, K. Takahashi, and Y. Yunoki, J. Phys. Soc. Jpn. 22, 219 (1967).

[17] K. Kroy and E. Frey, Phys. Rev. Lett. 77, 306 (1996).

[18] T. B. Liverpool, R. Golestanian, and K. Kremer, Phys. Rev. Lett. 80, 405 (1998).

[19] Jun-ichi Fukuda, Phys. Rev. E 59, 3275 (1999).

[20] Yu. B. Gaididei, P. L. Christiansen, K. Ф. Rasmussen, and M. Johansson, Phys. Rev. B 55, R13 365 (1997).

[21] M. Johansson, Yu. B. Gaididei, P. L. Christiansen, and K. Ф. Rasmussen, Phys. Rev. E 57, 4739 (1998).

[22] Yu. B. Gaididei, S. F. Mingaleev, P. L. Christiansen, and K. Ф. Rasmussen, Phys. Rev. E 55, 6141 (1997).

[23] J. C. Eilbeck, P. S. Lomdahl, and A. C. Scott, Physica D 16, 318 (1985).

[24] M. G. Vakhitov and A. A. Kolokolov, Izv. Vyssh. Uch. Zav. Radiofizika 16, 1020 (1973) [Radiophys. Quantum Electron. 16, 783 (1973)].

[25] J. J.-L. Ting and M. Peyrard, Phys. Rev. E 53, 1011 (1996).

[26] A. S. Davydov, Solitons in Molecular Systems (D. Reidel, Dordrecht, 1985).

[27] A. C. Scott, Phys. Rep. 217, 1 (1992).

[28] L. Cruzeiro-Hansson, Phys. Lett. A 249, 465 (1998).

[29] S. F. Mingaleev, P. L. Christiansen, Yu. B. Gaididei, M. Johanssaon, and K. Ф. Rasmussen, J. Biol. Phys. 25, 41 (1999). 